\title{
Characterization of multi-finger twist motion toward robotic rehabilitation
}

\author{
Reinhold Scherer, Sujata Pradhan, Brian Dellon, Daniel Kim, Roberta Klatzky, and Yoky Matsuoka
}

\begin{abstract}
Stroke or other neurological disorders potentially lead to movement deficits that may be recovered through proper rehabilitation. Robotic therapy has been applied to larger limbs such as the arms but not as commonly for the hand. It has been shown that virtual robotic environment with feedback distortion has a positive therapeutic effect in single finger movement. To expand this one finger therapeutic environment to more functional movements, our particular interest is applying feedback distortion to the everyday motor task of twisting bottle caps and jar lids. In order to understand what parameters to distort, unimpaired subjects' stereotypical movements were characterized with an intention to identify the differences with impaired population groups. This paper describes the hardware and software details of the haptic rotational twist-cap device developed to characterize, diagnose, and rehabilitate coordinated multi-finger twist movements. Using this device, it is shown the tangential and radial forces applied by each finger have stereotypical profiles for the unimpaired population. In addition, individual results from stroke, multiplesclerosis (MS), and elderly subject population are presented. This preliminary data show different types of deviation from the control data providing insight into parameters that should be distorted during robotic rehabilitation.
\end{abstract}

\section{INTRODUCTION}

Seventy percent of all patients have limited fine hand use in the first week, $41 \%$ at 3 months and $45 \%$ at 18 months after stroke [1]. Although there is evidence to the fact that vigorous early intervention after stroke may exacerbate brain damage [2] there is general consensus to the fact that early intervention after the patient is medically stable is ideal (after about 5-7 days after stroke) to promote neuroplasticity [3] and should include repetitive hand and finger motions [4]. There is also ample evidence that shows that incorporating functional tasks in rehabilitation protocols translates into far better outcomes [5] in comparison to uni-planar experimental tasks or tasks practiced in parts. Upper extremity functional performance is highly dependent on recovery of the hand due to the vast repertoire of movements contributing to function are dependent on dexterity of the hand [6] and thus this should be the part of training during recovery. Hand recovery has been a challenge partly due to the fact that the hand is

R. Scherer, D. Kim and Y.Matsuoka are with the Computer Science \& Engineering Department, University of Washington, Seattle, WA 98195, USA. schererdes. washington. edu

R. Scherer is with the Institute for Neurological Rehabilitation and Research, Rehabilitation center Judendorf-Strassengel, Judendorf-Strassengel, Austria.

S. Pradhan is with the Rehabilitation Medicine Department, University of Washington, Seattle, WA 98195, USA.

B. Dellon is with the Mechanical Engineering Department, Carnegie Mellon University, Pittsburgh, PA 15213, USA.

R. Klatzky is with the Psychology Department, Carnegie Mellon University, Pittsburgh, PA 15213, USA. affected to a greater degree to begin with, due to the extensive representation of the hand in the cortical homunculus, but also due to the fact that it is often neglected during rehabilitation. Conventional rehabilitation approaches utilizing exercise, orthotic support as well as electrical stimulation have shown that good recovery can be achieved in the hand in spite of some of the challenges associated with rehabilitation of the hand [7], [8], [9]. Despite strong evidence supporting this notion, research in rehabilitation after stroke using robotics has focused on training large-amplitude proximal joint movements, thus neglecting fine motor control related to the hand. Another major challenge for related technological developments is to provide engaging patient-tailored task oriented arm-hand training in natural environments with patient-tailored feedback to support (re)learning of motor skills [10]. Haptic devices are capable of not only providing realistic functional environments, but also providing focused and tailored feedback specific to individual requirements.

Some unique contributions of robotic therapy versus conventional therapies are that robots can be used as valuable adjuncts to save time and energy for the therapist, making rehabilitation sessions more efficient, and that rehabilitation protocols can be very precisely tailored to individual patients with only as much assistance provided as needed. The same protocols can also be used for objective assessment of improvement in status due to the capability of advanced sensing technologies available with the use of such devices. One of the important components of hand function is being able to control grasp during static conditions (holding objects without letting them slip) and under dynamic conditions (opening jars, transporting objects). Functionally relevant tasks have been shown to be of greater benefit for recovery after stroke [5].

Recently our group introduced the concept of feedback distortion, i.e., to introduce an undisclosed discrepancy between the actual movements and the virtual visual feedback the subjects receive about their movement [11], [12]. Feedback distortion targets individuals with chronic stroke and traumatic brain injury (TBI). These individuals have the capacity to make functional improvements; however, they may self-impose limits on their performance due to entrenched habits or psychological barriers [11]. Previously, a single finger therapeutic paradigm has been implemented using visual distortion in the virtual robotic environment [13]. This study has shown the finger's range of motion to increase by more than $50 \%$, a spasticity measure (Ashworth score) to be reduced, and functional scores (such as the AMAT score) to improve after 6 weeks of therapy for 


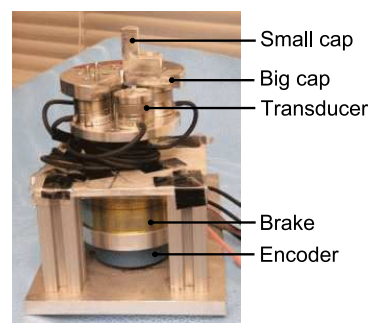

(a)

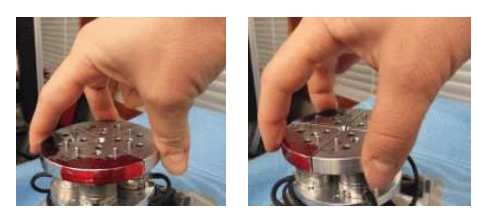

(c)

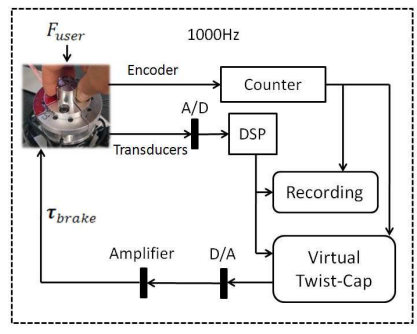

(b)

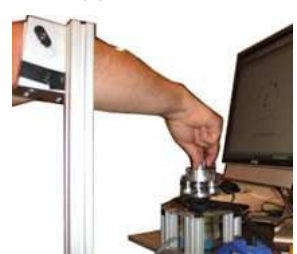

(d)
Fig. 1. Twist-cap device. (a) Picture of the twist-cap device intended to characterize, diagnose, and rehabilitate functional twisting movement. Here, one of the quadrant's small cap pieces is left on to demonstrate that these pieces can be added to make different size caps. (b) Diagram of the measurement setup. (c). Finger positions for THI (left) where the index finger and the thumb are opposed to each other, and THM (right) where the middle finger and the thumb are opposed to each other. (d) Picture of the experimental set-up showing the elbow placed on the elbow-rest, the twist-cap device and a screen providing visual feedback (virtual cap).

subjects 2-8 years post injury. We have also shown that these improvements can be retained [14]. While this study has shown promise for the use of virtual robotic environment and the distorted feedback, it has worked with only one finger movemetnt at a time. In order to translate these applications into more coordinated movements that will have functional significance in the outcome, we aim to apply the similar distorted feedback paradigm for a multi-finger task. Specifically we chose the cap-twisting motion because these movements are heavily used in our daily tasks and is a simple extension of a single finger movement. Even with a simple three-finger twist movement, the total number of parameters potential for distortion suddenly explodes. For example, we can distort the finger position, force, force balance/coordinate between fingers, radial/tangential force balance, etc. Therefore, in order to understand what to distort to improve the function, we must first understand what the stereotypical movements are like in healthy young subjects and compare the differences with different subject groups who have difficult time producing the twisting motion.

In this paper, we describe the hardware and software details of the haptic rotational twist-cap device developed to characterize, diagnose, and rehabilitate coordinated multifinger twist movements and present normative data of a younger able-bodied subject group. We also have a small set of preliminary results from stroke, multiple-sclerosis (MS), and elderly subject population.

\section{METHODS}

\section{A. TWIST-CAP DEVICE}

The twist-cap device consists of four 6 DOF force/torque transducers (ATI Industrial Automation, Nano25) patterned radially about a central axis [15]. One side of the transducers is rigidly fixed to the rotor of a magnetic particle brake (Placid Industries, B15) as shown in Fig.1(a), which is driven by a linear amplifier (Trust Automation, TA105). The other side of each transducer is covered by circular quadrants, which together form the virtual twist-cap. Small gaps between each quadrant allow the transducer to detect an individual finger's motion exclusive of other digits force production. Smaller quadrants can be affixed on top of the transducers to provide different cap sizes. The rotation angle was measured by using an optical quadrature encoder (Agilent Technologies, HEDS-6540/T13) with a resolution of $0.18^{\circ}$ (Fig.1.a).

Fig.1.b shows a diagram of the measurement set-up. The force/torque signals and the encoder output were digitized with a National Instruments data acquisition board (6071E) at a rate of $1000 \mathrm{~Hz}$, and then stored to disk. Additionally real-time feedback on the cap position was presented on the screen (virtual cap). The device also has the capability to provide real-time feedback of the force/torques. Signal conditioning can be found in II-D.1. The software was implemented by using Matlab/Simulink (The MathWorks, Inc., Natick, MA, USA) and the Real Time Windows Target toolbox.

\section{B. EXPERIMENTAL PARADIGM}

To characterize twisting strategies, we recorded data from three fingers during different experimental conditions. These were:

- Two finger positions. Either the index finger (condition THI) or the middle finger (condition THM) was opposite to the thumb. The third finger was placed on the intermediate position (Fig.1(c)).

- Two cap sizes. The small cap had a radius $R=12 \mathrm{~mm}$ (condition SML) and the large cap size had $R=44 \mathrm{~mm}$ (condition LRG).

- Two twist directions. Clock-wise (condition CLW) or counter-clockwise (condition CCW).

- Two force levels based on the maximum force production level which was measured during the calibration phase. The level was either $40 \%$ (condition F40) or $60 \%$ (condition F60) of the maximum voluntary force level.

The experiment consisted of four sets of 20 trials each. Alternately experiments started either with the large (LRG) or small (SML) cap and with finger position THI or THM. For each trial subjects had the task of twisting the cap according to the finger position and direction (CLW vs. $\mathrm{CCW}$ ) presented on the screen. The rotational movement was subdivided into two 90 degree twists, each requiring the subject to grasp the cap, perform a 90 degree twist and release the cap. After the first 90 degree twist the screen was blanked for $500 \mathrm{~ms}$ before the go signal for the second twist was shown. The direction of the twists was alternating from trial to trial (random at the beginning). The force levels F40 and F60 were randomly distributed over the trials. To avoid arm or body usage and to ensure that finger and wrist were used to perform the motor task, the right elbow was 
placed on an elbow-rest (Fig.1(d)). No feedback distortion was presented to subjects.

The maximum comfortable force production level $F_{M A X}$ for each subject was measured individually for conditions SML and BIG. Subjects were told to produce a constant maximum twisting force that could be comfortably maintained for $3 \mathrm{~s}$. $F_{M A X}$ was defined as the average force of the 3-s period.

\section{SUBJECTS}

Thirteen right-handed volunteers participated in the twistcap experiment:

- Ten young able-bodied individuals $(24.9 \pm 6.6 \mathrm{yr}, 8$ male, 2 female) constituted the control group. All were without any medical or psychological disease and had normal or corrected to normal vision.

- One 64 yr old woman was a stroke age-matched group. She was a spectacle wearer and under medical treatment for arthritis. This subject did not feel comfortable twisting the large cap under finger condition THM.

- One 52 yr old man with multiple sclerosis (MS) affecting primarily his sensory feedback.

- One 88 yr old man who had a stroke in the year 2000. He was able to manipulate objects with his right hand, however, he had problems turning on a water tap or opening soda bottles. Furthermore, since his episode the patient was sensitive to pain, especially in his legs and index finger. To manage pain, we did not conduct (THI, BIG) and (THM, SML) conditions.

All subjects gave informed consent after the aim of the study and the experimental procedure had been explained to them. The study had been reviewed and approved by the University of Washington institutional review board.

\section{DATA ANALYSIS}

1) SIGNAL CONDITIONG : At the beginning of each experiment $10 \mathrm{~s}$ baseline data were collected. During this period subjects were not touching the twist-cap. By subtracting the average value of the baseline and by multiplication with the transducer calibration matrix, the recorded signals were converted into forces/torques. A $5^{\text {th }}$ order Butterworth lowpass filter with cut-off frequency at $8 \mathrm{~Hz}$ was was used to smooth the data.

2) RADIAL AND TANGENTIAL FORCES: We are interested in the radial $\left(F_{r a d}\right)$ and tangential $\left(F_{t a n}\right)$ forces applied to each quadrant of the cap from thumb, index and middle finger. To compute $F_{\text {rad }}$ and $F_{t a n}$ we need to identify the position of the finger on the twist cap quadrant. Fig.2 illustrates this problem. Let $\vec{F}$ denote the force acting at point $\vec{P}_{3}$ of the quadrant (radius $R$ ). Operating under the assumption that inertial interactions are negligible, a static analysis can be used to solve for $\vec{P}_{3}$ given the transducer reactions, $F_{x}, F_{y}$, and torque $M_{z}$. First a normal is defined relative to the orientation of the transducer's force reaction,

$$
\hat{F}_{\perp}=\vec{F} \times \hat{Z}
$$

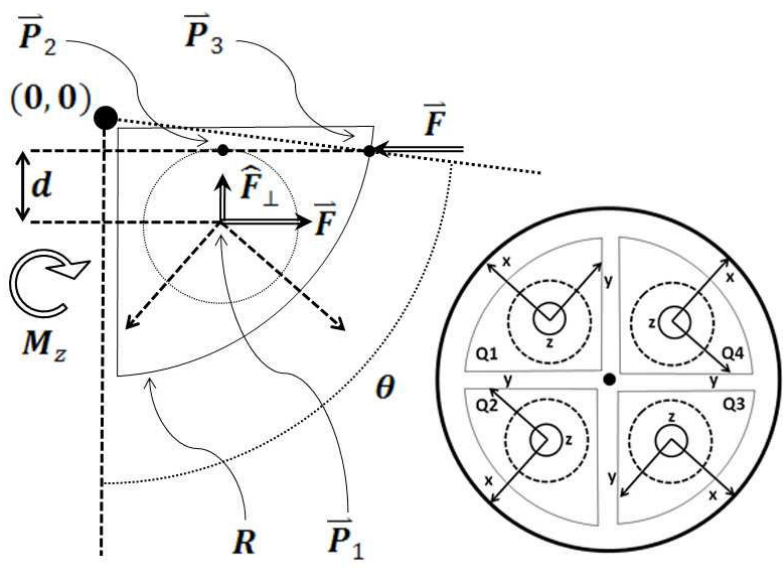

Fig. 2. Cartoon illustrating the computation of the finger position on the cap (angle $\Theta$ ). The scheme on the right side shows the axes of the transducers (planview).

Where $\hat{Z}$ is a unit vector along z. Due to the statics of the situation we can solve for the perpendicular moment arm of the finger force about the transducer. The length of the lever arm $d$ can be computed according to,

$$
d=M_{z} /\|F\|
$$

A point on the finger force's line of action can now be found by traveling the distance $d$ along the vector $\hat{F}_{\perp}$ starting from the transducer's center, $\vec{P}_{1}$. This results in the point, $\vec{P}_{2}$,

$$
\overrightarrow{P_{2}}=\overrightarrow{P_{1}}+d \cdot \hat{F}_{N}
$$

The direction of finger force is already known, $\hat{F}$, therefore the final step to locate $\vec{P}_{3}$ is to determine the intersection of the force's line of action through $\vec{P}_{2}$ with the quadrants perimeter. The equation representing this condition is given by,

$$
\left|\overrightarrow{P_{2}}+t \cdot \hat{F}\right|=R
$$

This equation can be expanded to form a quadratic in the parameter $t$. Solving for $t$, and plugging back into equation (4) results in $\vec{P}_{3}$. From $\vec{P}_{3}$, angle $\Theta$ can easily be generated using the arctangent. Finally the finger force can be decomposed, correctly, into $F_{r a d}$ and $F_{t a n}$ using the following transformation,

$$
\begin{gathered}
F_{\text {rad }}=\left(\vec{F} \bullet \hat{P}_{3}\right) \cdot \hat{P}_{3} \\
F_{\text {tan }}=\vec{F}-F_{\text {rad }} .
\end{gathered}
$$

3) NORMALIZATION: In order to compare data across subjects, the computed time series were normalized. Trials were subdivided into 2 periods of interest, the pre-twisting $\left(T_{\text {pre }}\right)$ and the twisting period $\left(T_{\text {twist }}\right) . T_{\text {pre }}$ period was defined as the time when the finger touch was first detected $\left(t_{\text {touch }}\right)$ and when the cap started to rotate for the first $5^{\circ}$ $\left(t_{5^{\circ}}\right)$. The $t_{\text {touch }}$ was defined as when $F_{\text {rad }}$ exceeded two standard deviations from the mean $F_{r a d}$ after the go signal was presented to the subject. For each trial the $T_{\text {pre }}$ period was normalized by time ( $t_{\text {touch }}$ at 0 and $t_{5^{\circ}}$ at 1$)$ and the 

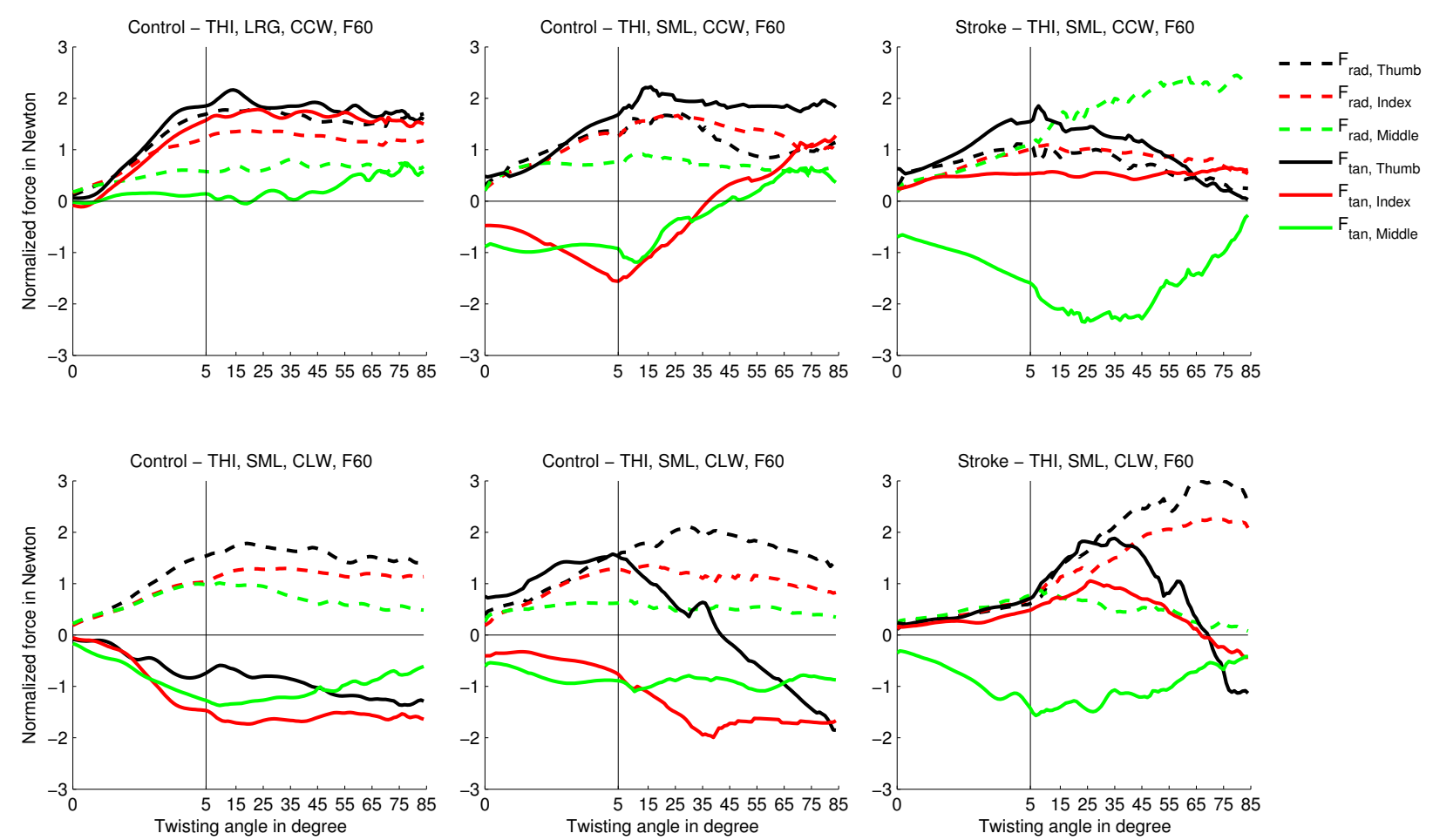

Fig. 3. Average $F_{r a d}$ and $F_{t a n}$ time course for thumb, index and middle finger for one typical control subject (column 1-2) and the stroke patient (column 3) for different conditions. $F_{\text {rad }}$ toward the center of the cap are positive and thus all $F_{\text {rad }}$ are positive. Positive $F_{\text {tan }}$ contribute to CCW movement and negative $F_{t a n}$ contribute to CLW movement. The vertical line in each plot marks the border between $T_{p r e}$ and $T_{t w i s t}$.

$T_{\text {twist }}$ period by the twist cap angle between $5^{\circ}$ and $85^{\circ}$. The average of the normalized trials was computed and rescaled by dividing the individual forces of each finger by the sum of forces applied by all three finger. $F_{\text {rad }}$ and $F_{\text {tan }}$ were rescaled individually.

4) IDENTIFICATION OF MOVEMENT PATTERNS: In order to find characteristic parameters across the control group, we computed correlations for different combinations of conditions. For each of the following three condition sets eight combinations, respectively, were analyzed: (finger position, cap size, twist direction), (finger position, cap size, force level) and (finger position, force level, twist direction). For each set the average value, the linear slope and the quadratic slope of the normalized $F_{\text {rad }}$ and $F_{\text {tan }}$ within $T_{\text {pre }}$ and $T_{\text {twist }}$ were investigated individually. For example for the combination (THI, SML, CLW) and parameter average $F_{\text {rad }}$, the average $F_{\text {rad }}$ at force level F40 and F60 for thumb, index and middle finger were used to model the twist motion (6 parameters for each condition). To find characteristic movement patterns principle components analysis (PCA) was performed with the most correlated parameter for all combinations.

\section{RESULTS}

For $T_{\text {pre }}$ and $T_{\text {twist }}$ independently the average $F_{\text {rad }}$ and $F_{t a n}$ were most correlated across all combination of conditions (average Pearson correlation coefficient 0.75). Our investigation therefore focused on this parameter.

Fig.3 shows example time courses of $F_{r a d}$ and $F_{\text {tan }}$ for thumb, index and middle finger for conditions (THI, LRG, CLW, F60), (THI, LRG, CCW, F60), (THI, SML, CLW, F60) and (THI, SML, CCW, F60) for one typical control subject. Additionally results of the stroke patient are shown. $F_{\text {rad }}$ toward the center of the cap is positive and thus all $F_{\text {rad }}$ recorded during the trials are positive. Positive $F_{\text {tan }}$ contribute to $\mathrm{CCW}$ movement and negative $F_{\text {tan }}$ contribute to CLW movement. For the control subject the curves illustrate an increase of $F_{\text {rad }}$ in $T_{\text {pre }}$ followed by a steady or decrease in magnitude during $T_{\text {twist }}$. The stroke patient in contrast shows an increase of $F_{\text {rad }}$ also during the latter. $F_{\text {tan }}$ during the $T_{\text {twist }}$ are characteristic for the direction of the twist motion for LRG. However, notice that one or more fingers produce a $F_{t a n}$ that is against the movement direction in some cases. The index/middle finger for CCW and thumb for CLW for SML are acting contrary to the twisting direction in the first third of the motion. This antagonist pattern is visible also for the stroke patient.

The average $F_{\text {rad }}$ and $F_{\text {tan }}$ for $T_{\text {pre }}$ and $T_{\text {twist }}$ are summarized in Fig. 4 for all subjects. The plots show consistent force patterns for the control group for each finger. Paired t-tests confirm a significant increase from $F_{\text {rad }}\left(T_{\text {pre }}\right)$ to $F_{\text {rad }}\left(T_{\text {twist }}\right)$ for thumb (THI: $0.96 \pm 0.13$ vs. $1.42 \pm 0.21$, $\mathrm{p}=0.0001$; THM: $0.94 \pm 0.14$ vs. $1.34 \pm 0.26, \mathrm{p}=0.0001)$, index finger (THI: $0.77 \pm 0.13$ vs. $1.14 \pm 0.22, \mathrm{p}=0.0001$; THM: 

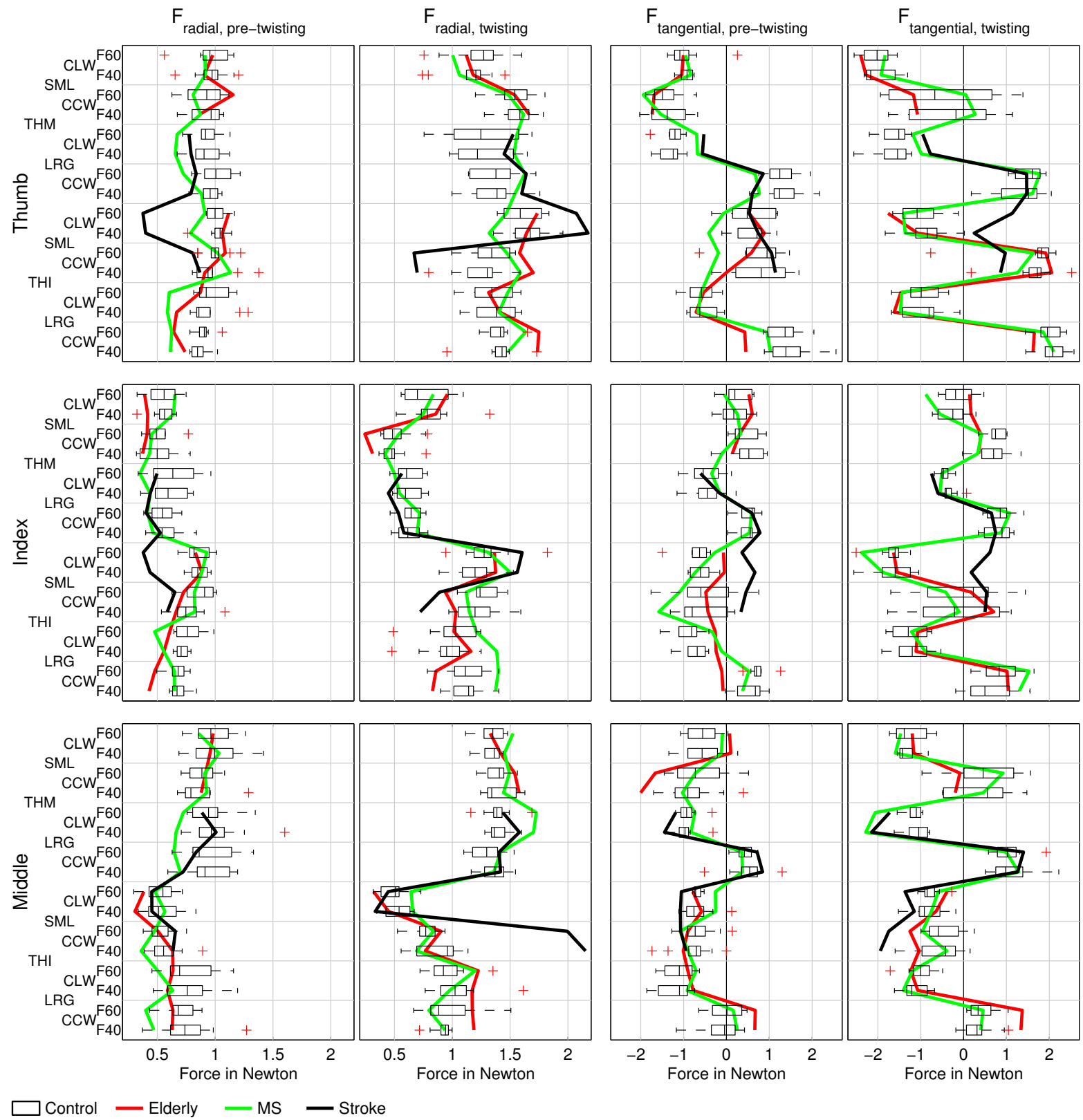

Fig. 4. $\quad F_{\text {rad }}$ and $F_{t a n}$ during $T_{\text {pre }}$ and $T_{\text {twist }}$. The boxplots show the distribution of the control group. The boxes have lines at the $25^{t h}$ percentile, median, and $75^{t h}$ percentile. The lines extending from each end of the boxes (whiskers) show the extent of the rest of the data. Outliers, i.e., values beyond the end of the whiskers, are marked with the '+' symbol. Superimposed are the values for the elderly, MS and stroke.

$0.56 \pm 0.15$ vs. $0.64 \pm 0.17, \mathrm{p}=0.0016)$ and middle finger (THI: $0.64 \pm 0.22$ vs. $0.81 \pm 0.26, \mathrm{p}=0.0001$; THM: $0.94 \pm 0.20$ vs. $1.364 \pm 0.12, \mathrm{p}=0.0001)$. The correlation of $F_{t a n}\left(T_{t w i s t}\right)$ of thumb, index and middle finger with the direction of twist motion was $0.93,0.79$ and 0.65 , respectively, for THI; corresponding correlations for THM were $0.78,0.87$ and 0.88 , respectively.

The average $F_{\text {tan }}$ of the elderly subject (Fig.4) and of the individual with MS were within the distribution of the control group. For both individuals $F_{\text {rad }}$ during $T_{\text {twist }}$ were close to either the lower or the upper border of the distribution of the control group. The stroke patient showed a different pattern. $F_{\text {rad }}$ and $F_{t a n}$ were beyond the bounds of the control group. While there was no correlation found between $F_{\text {rad }}$ and $F_{t a n}$ for the control group (correlation coefficient $<0.2$ ), the stroke subject showed a correlation $(0.78)$ between $F_{\text {rad }}$ and $F_{\text {tan }}$ during $T_{\text {twist }}$.

Two principal components were required to represent minimum (average over conditions) $68 \%(74 \%)$ of $F_{\text {rad }}$ and $74 \%(76 \%)$ of $F_{\text {tand }}$ during $T_{\text {pre }}$. During $T_{\text {twist }}$ two principal components represented $72 \%(79 \%)$ of $F_{\text {rad }}$ and $72 \%(91 \%)$ of $F_{t a n}$. Tab.I shows principal components for $F_{t a n}$ for conditions (THI, SML, CCW) and (THM, SML, CCW). In both cases two components account for about $80 \%$ of the 
TABLE I

PRINCIPAL COMPONENTS FOR $F_{\text {tan }}$.

\begin{tabular}{ccrr} 
& & \multicolumn{2}{c}{ Components } \\
Condition & Finger & 1 & 2 \\
\hline THI, SML, CCW, F40 & Thumb & $\mathbf{0 . 9 1 9}$ & 0.106 \\
& Index & $\mathbf{0 . 8 8 8}$ & -0.330 \\
& Middle & 0.254 & $\mathbf{0 . 8 8 5}$ \\
THI, SML, CCW, F60 & Thumb & $\mathbf{0 . 9 2 9}$ & 0.107 \\
& Index & $\mathbf{0 . 8 7 7}$ & -0.281 \\
& Middle & 0.147 & $\mathbf{0 . 7 9 9}$ \\
\hline Cumulative \% & & $55.9 \%$ & $83.1 \%$ \\
\hline THM, SML, CCW, F40 & Thumb & 0.473 & $\mathbf{0 . 7 6 1}$ \\
& Index & $\mathbf{0 . 8 7 2}$ & -0.376 \\
THM, SML, CCW, F40 & Middle & $\mathbf{0 . 9 0 2}$ & -0.279 \\
& Thumb & 0.192 & $\mathbf{0 . 9 5 2}$ \\
& Index & $\mathbf{0 . 9 2 4}$ & -0.160 \\
& Middle & 0.409 & 0.451 \\
\hline Cumulative \% & & $47.6 \%$ & $79.8 \%$ \\
\hline
\end{tabular}

variance in the data. These results are consistent with the pattern in Fig.4.

\section{DISCUSSION}

The control group results showed that subjects, in general, use a consistent strategy. There are a few characteristic parameters while making these movements. First, the finger that was opposing the thumb contributes more in radial force and twisting than the finger that is 90 degrees off from the thumb. Second, the thumb contributes the most in twisting when the index finger is opposed (THI), while the thumb contributes the least in twisting when the middle finger is opposed (THM).

Another interesting pattern is shown in Fig.3. The curve suggests that, depending on the twist direction and the relationship of the geometry of the cap and the hand posture, individual fingers produce forces which act against the twist direction. We believe that this opposite force contribution comes from the thumb kinematics that makes it difficult to produce assisting force for twisting in those configurations. The curves in Fig. 3 and more clearly the distribution in Fig.4 show that the thumb produces highest radial forces for condition (THI, SML, CLW). The reason for this is that the force acts along the trajectory/axis of the thumb.

The $F_{t a n}$ of the elderly individual was within the range of the control group. Due to the arthritis the elderly subject could not stress the index finger. Consequently thumb and middle finger had to apply higher forces. For the MS patient the pattern suggest an increased radial forces for all fingers. This behavior may arise due to the loss of somatosensory feedback [16].

The chronic stroke patient showed a different pattern. The radial forces for the small cap were much higher compared to all other individuals. Furthermore, for this individual radial and tangential forces were correlated. One explanation for this behavior might be the pain the patient was experiencing. It may also be related to the disruption in the coordinated movement among fingers.

The preliminary results in this study suggest that radial and tangential forces can be used to characterize twist motion patterns. This is in accordance with the literature [17]. Furthermore, the force profile of the stroke patient suggests that these two parameter are suited for distortion feedback therapy.

Some of the described effects may be caused by the constraints imposed from the experimental paradigm. In real life people potentially use different strategies, e.g., the arm as lever to open a water bottle or the palm of the hand to open a jar. The set-up, however, allows the study of patterns of individual fingers and the interaction of multiple fingers.

The introduced twist-cap device is a small, compact robot able to provide haptic feedback without actuators. The brake is a passive mechanical element which makes the device safe and thus also suitable for home application. The system can be used to train the multi-finger and wrist coordination, strength, and pinching/twisting strategies.

\section{REFERENCES}

[1] A.K. Welmer, L.W. Holmqvist, D.K., and Sommerfeld, Limited fine hand use after stroke and its association with other disabilities, $J$ Rehabil Med vol 40(8), 2008, pp. 603-8.

[2] A. Risedal, J. Zeng and B.B. Johansson, Early training may exacerbate brain damage after focal brain ischemia in the rat, J Cereb Blood Flow Metab, vol 19(9), 1999, pp. 997-1003.

[3] A. Turton and V. Pomeroy. When should upper limb function be trained after stroke? Evidence for and against early intervention, NeuroRehabilitation, vol 17(3), 2002, 215-24.

[4] Petr Hlustik and Michal Mayer, Paretic hand in stroke: from motor cortical plasticity research to rehabilitation, Cogn Behav Neurol, vol 19(1), 2006, pp. 34-40.

[5] Jan Z Davis, Task selection and enriched environments: a functional upper extremity training program for stroke survivors, Top Stroke Rehabil, vol 13(3), 2006, pp. 1-11.

[6] M. Kimmerle, L. Mainwaring and M. Borenstein, The functional repertoire of the hand and its application to assessment, Am J Occup Ther, vol 57, 2003, pp. 489-498.

[7] D.G. Pitts and S.P. O'Brien, Splinting the hand to enhance motor control and brain plasticity, Top Stroke Rehabil, vol 15(5), 2008, pp. 456-67.

[8] V. Gritsenko and A. Prochazka, A functional electric stimulationassisted exercise therapy system for hemiplegic hand function, Arch Phys Med Rehabil, vol 85(6), 2004, pp. 881-5.

[9] E. Bürge, D. Kupper, A. Finckh, S. Ryerson, A. Schnider, and B. Leemann, Neutral functional realignment orthosis prevents hand pain in patients with subacute stroke: a randomized trial, Arch Phys Med Rehabil, vol 89(10), 2008, pp. 1857-62.

[10] A.A. Timmermans, H.A. Seelen, R.D. Willmann, and H. Kingma, Technology-assisted training of arm-hand skills in stroke: concepts on reacquisition of motor control and therapist guidelines for rehabilitation technology design, J Neuroeng Rehabil col 6(1), 2009, Epub.

[11] B.R. Brewer, M. Fagan, R.L. Klatzky, and Y. Matsuoka, Perceptual limits for a robotic rehabilitation environment using visual feedback distortion, IEEE Trans Neural Syst Rehabil Eng, vol. 13(1), 2005, pp. $1-11$.

[12] B. Dellon and Y. Matsuoka, Feedback Distortion to Augment Controllability of Human Limb Motion, Proceedings of Virtual Rehabilitation, 2008.

[13] B.R. Brewer, R. Klatzky, and Y. Matsuoka, Visual Feedback Distortion in a Robotic Rehabilitation Environment, The Proceedings of IEEE, vol. 94(9), 2006, pp. 1739-1751.

[14] B. Brewer, R. Klatzky, and Y. Matsuoka, Visual feedback distortion in a robotic environment for hand rehabilitation, Brain Research Bulletin, vol 75*6), 2008, pp. 804-13.

[15] G. Chu. Twist cap device. Undergraduate thesis, Mechanical Engineering, Carnegie Mellon University, 2005.

[16] A.G. Witney, A. Wing, J.L.. Thonnard, and A.M. Smith, The cutaneous contribution to adaptive precision grip, Trends Neurosci, vol. 27(10), 2004, pp. 637-43.

[17] V.M.Zatsiorsky, and M.L. Latash, Multifinger prehension: an overview, J Mot Behav, vol 40(5), 2008, pp. 446-76. 